\title{
Cut-Elimination in the Intuitionistic Many-Valued Logic Based \\ on a Partial Order
}

\author{
Mitio Takano \\ Nïgata University
}

The purpose of this paper is to prove the following cut-elimination theorem for the intuitionistic many-valued logic based on a partial order, which was claimed in Takano [1, Theorem 14]:

Theorem. Assume that ( $T$, $\leqq)$ forms a lower semi-lattice. Then every provable matrix is cut-free provable provided that it is permissible.

Readers are supposed to be familiar with our previous paper [1].

$\S 1$. Recall that a matrix $K$ is permissible iff for every formula $A$ the following conditions $\mathbf{P} 1[A]$ and $\mathbf{P} 2[A]$ hold :

$\mathbf{P 1}[A]$. If $\langle\lambda, A\rangle \in K$, then either $\lambda^{-} \times\{A\} \subseteq K$ or $\lambda^{+} \times\{A\} \subseteq K$.

P2 $[A]$. If the set $\{\lambda \mid\langle\lambda, A\rangle \notin K\}$ is not empty, then it has the least element $\min \{\lambda \mid\langle\lambda, A\rangle \notin K\}$.

We show some simple properties of permissible matrices, which we shall use without mention.

Proposition 1. Suppose $\lambda_{0}=\min \{\lambda \mid\langle\lambda, A\rangle \notin K\}$. Then $\lambda_{0} \leqq \mu$ if and only if $\urcorner\left[\mu^{-} \times\{A\} \subseteq K\right]$.

Proof. If $\lambda_{0} \leqq \mu$, then $\left\langle\lambda_{0}, A\right\rangle$ belongs to $\mu^{-} \times\{A\}$ but does not to $K$, so $\rceil\left[\mu^{-}\right.$ $\times\{A\} \subseteq K]$. To prove the converse, suppose $\rceil\left[\mu^{-} \times\{A\} \subseteq K\right]$. Then $\lambda \leqq \mu$ but $<\lambda$, $A\rangle \notin K$ for some $\lambda$. Hence $\lambda_{0} \leqq \lambda \leqq \mu$, so $\lambda_{0} \leqq \mu$.

Proposition 2. Suppose $\lambda_{0}=\min \{\lambda \mid\langle\lambda, A\rangle \notin K\}$ and $\lambda_{0} \in U \subseteq T$. Then $(K$ $\cup(U \times\{A\}))^{\circ}=K^{\circ}$.

Proof. It suffices to prove that $\langle\mu, A\rangle \in K^{\circ}$ whenever $\langle\mu, A\rangle \in(K \cup(U \times$ $\{A\}))^{\circ}$. If $\langle\mu, A\rangle \in(K \cup(U \times\{A\}))^{\circ}$, that is, if $\mu^{-} \times\{A\} \subseteq K \cup(U \times\{A\})$, then \rceil$\left(\lambda_{0} \leqq \mu\right)$ since $\left\langle\lambda_{0}, A\right\rangle \notin K \cup(U \times\{A\})$, so $\mu^{-} \times\{A\} \subseteq K$, that is, $\langle\mu, A\rangle \in K^{\circ}$.

Proposition 3. $\left.1^{\circ}\right)$ Assume that $(T, \leqq)$ forms a lower semi-lattice. Then the empty matrix $\emptyset$ is permissible.

$\left.2^{\circ}\right)$ If $K$ is permissible, so are $K^{\circ}, K \cup\left(\mu^{\wedge} \times\{A\}\right)$ and $K \cup\left(\mu^{+} \times\{A\}\right)$.

Proof. $1^{\circ}$ ) By the assumption $T$ has the least element $\min (T)$, and $\min (T)$

Annals of the Japan Association for Philosophy of Science, March 1988 
$=\min \{\lambda \mid\langle\lambda, B\rangle \notin \emptyset\}$.

$\left.2^{\circ}\right)$ Suppose that $K$ is permissible. If $\langle\lambda, B\rangle \in K^{\circ}$, then $\lambda^{-} \times\{B\} \subseteq K^{\circ}$; on the other hand, if $\left\{\lambda \mid\langle\lambda, B\rangle \notin K^{\circ}\right\} \neq \emptyset$, then $\{\lambda \mid\langle\lambda, B\rangle \in K\} \neq \emptyset$ and

$$
\min \{\lambda \mid\langle\lambda, B\rangle \notin K\}=\min \left\{\lambda \mid\langle\lambda, B\rangle \notin K^{\circ}\right\} ;
$$

so $K^{\circ}$ is permissible. If $\lambda \in \mu^{\wedge}$, that is, if $\lambda \neq \mu$, then $\lambda^{+} \subseteq \mu^{\wedge}$ or $\lambda^{-} \subseteq \mu^{\wedge}$ according as $7(\lambda \leqq \mu)$ or $\rceil(\mu \leqq \lambda)$; on the other hand, if $\left\{\lambda \mid\langle\lambda, A\rangle \notin K \cup\left(\mu^{\wedge} \times\right.\right.$ $\{A\})\} \neq \emptyset$, then $\mu=\min \left\{\lambda \mid\langle\lambda, A\rangle \notin K \cup\left(\mu^{\wedge} \times\{A\}\right)\right\}$; so $K \cup\left(\mu^{\wedge} \times\{A\}\right)$ is permissible. If $\lambda \in \mu^{+}$, then $\lambda^{+} \subseteq \mu^{+}$; on the other hand, if $\left\{\lambda \mid\langle\lambda, A\rangle \notin K \cup\left(\mu^{+} \times\{A\}\right)\right\}$ $\neq \emptyset$, then $\{\lambda \mid\langle\lambda, A\rangle \notin K\} \neq \emptyset$ and

$$
\min \{\lambda \mid\langle\lambda, A\rangle \notin K\}=\min \left\{\lambda \mid\langle\lambda, A\rangle \notin K \cup\left(\mu^{+} \times\{A\}\right)\right\} ;
$$

so $K \cup\left(\mu^{+} \times\{A\}\right)$ is also permissible.

§ 2. In this section we shall explain that, to prove Theorem it suffices to show the following lemma :

Lemma. Assume that $(T, \leqq)$ forms a lower semi-lattice. If $K$ is permissible, $\mapsto K \cup\left(U_{1} \times\{A\}\right), \mapsto K \cup\left(U_{2} \times\{A\}\right)$ and if $\left(U_{1}, U_{2}\right)$ is a pair of disjoint subsets of $T$, then $\rightarrow K$.

Recall that the cut inference has the following form:

Cut inference :

$$
\frac{K \cup\{\langle\mu, A\rangle\} K \cup\{\langle\nu, A\rangle\}}{K}
$$

where $\mu \neq \nu$.

If we replace the cut inference by the new inference rule described below, we obtain another formal system from one developed in $[1, \S 4]$ :

Cut inference in the second form:

$$
\frac{K \cup\left(\mu^{\wedge} \times\{A\}\right) \text { for every } \mu \text { in } T}{K} .
$$

Let $\vdash^{*} K$ denote that $K$ is provable in the new system.

Proposition 4. If $\vdash K$, then $\vdash^{*} K$.

Proor. It suffices to prove that, if $\vdash^{*} L \cup\left\{\left\langle\lambda_{1}, A\right\rangle\right\}, \vdash^{*} L \cup\left\{\left\langle\lambda_{2}, A\right\rangle\right\}$, and if $\lambda_{1} \neq \lambda_{2}$, then $\vdash^{*} L$. Suppose $\vdash^{*} L \cup\left\{\left\langle\lambda_{1}, A\right\rangle\right\}, \vdash^{*} L \cup\left\{\left\langle\lambda_{2}, A\right\rangle\right\}$ and $\lambda_{1} \neq \lambda_{2}$. For every $\mu$ in $T$, either $\mu \neq \lambda_{1}$ or $\mu \neq \lambda_{2}$, that is, either $\lambda_{1} \in \mu^{\wedge}$ or $\lambda_{2} \in \mu^{\wedge}$, so in view of the fact that

$$
\vdash^{*} L_{2} \text {, whenever } \vdash^{*} L_{1} \text { and } L_{1} \subseteq L_{2} \text {, }
$$

we obtain $\vdash^{*} L \cup\left(\mu^{\wedge} \times\{A\}\right)$ in either case. Hence by the cut inference in the second form, we have $\vdash^{*} L$.

Proposition 5. Suppose that $\mathfrak{B}$ is a proof-figure of the new system. If the 
end-matrix of $\mathfrak{B}$ is permissible, so are all matrices contained in it.

Proor. If the conclusion of the cut inference in the second form is permissible, so are premises. Similarly for left or right inferences.

Proposition 6. Assume that a formula $B$ is given. Assume further that for every permissible matrix $K$ and for every pair $(U, V)$ of disjoint subsets of $T$, if $\mapsto \rightarrow$ $K \cup(U \times\{B\})$ and $\mid \rightarrow K \cup(V \times\{B\})$, then $\rightarrow K$. If $L$ is permissible and if $\rightarrow L$ $\cup\left(\mu^{\wedge} \times\{B\}\right)$ for every $\mu$ in $T$, then $\rightarrow L$.

Proof. We put $L(S)=L \cup(S \times\{B\})$ and $n(S)=$ Card $\{\lambda \mid\langle\lambda, B\rangle \notin L(S)\}$ for every subset $S$ of $T$, where $\operatorname{Card}(W)$ denotes the cardinality of the set $W$. Suppose that $L$ is permissible and $\mid \rightarrow L\left(\mu^{\wedge}\right)$ for every $\mu$. To show $\rightarrow L$, that is, to show $\rightarrow$ $L(\emptyset)$, it suffices to prove that $L(S)$ is cut-free provable whenever it is permissible, which we shall prove by induction on $n(S)$.

CASE 1. $n(S)=0 . \quad L(S)$ is basic since it contains $T \times\{B\}$, so $\rightarrow L(S)$. $L(S)$.

CASE 2. $n(S)=1$. Since $L(S)=L\left(\mu^{\wedge}\right)$ for some $\mu$, by the assumption $\rightarrow$

CASE 3. Otherwise. In view of the fact that $L(S)$ is permissible, we put $\lambda_{0}=$ $\min \{\lambda \mid\langle\lambda, B\rangle \notin L(S)\}$. We take $\lambda_{1}$ such that $\left\langle\lambda_{1}, B\right\rangle \notin L(S)$ and $\lambda_{1} \neq \lambda_{0}$. Then both $L(S) \cup\left(\lambda_{1}{ }^{+} \times\{B\}\right)$ and $L(S) \cup\left(\left(T-\lambda_{1}{ }^{+}\right) \times\{B\}\right)$ are permissible; the latter claim follows from the facts that $\lambda^{-} \subseteq T-\lambda_{1}{ }^{+}$whenever $\lambda \in T-\lambda_{1}{ }^{+}$and that

$$
\lambda_{1}=\min \left\{\lambda \mid\langle\lambda, B\rangle \notin L(S) \cup\left(\left(T-\lambda_{1}{ }^{+}\right) \times\{B\}\right)\right\} .
$$

Hence by the induction hypothesis they are cut-free provable, so by the assumption of the proposition, we have $\rightarrow L(S)$.

Proof that Lemma imples Theorem. Suppose that Lemma holds. Suppose further that a permissible matrix $K$ is provable. Then $\vdash^{*} K$ by Proposition 4, so we let $\mathfrak{B}$ be the proof-figure of the new system having $K$ as its end-matrix. By Proposition 5, every matrices contained in $\mathfrak{B}$ is permissible. Hence, in view of the fact that

(2) $\rightarrow L$, whenever $L$ is permissible and $\rightarrow L \cup\left(\mu^{\wedge} \times\{B\}\right)$ for every $\mu$ in $T$, which follows from Lemma and Proposition 6, each matrices contained in $\mathfrak{P}$ is cut-free provable; especially $\mid \rightarrow K$.

§3. The purpose of this section is to prove Lemma. It suffices to show, on the assumption of Lemma, that if $K$ is permissible, $\left|\rightarrow{ }_{n_{1}} K \cup\left(U_{1} \times\{A\}\right),\right| \rightarrow{ }_{n_{2}} K \cup\left(U_{2}\right.$ $\times\{A\})$ and if $U_{1} \cap U_{2}=\emptyset$, then $\rightarrow K$, which we shall prove by induction on $\omega \cdot$ $d(A)+n_{1}+n_{2}$, where $d(A)$ denotes the number of occurrences of connectives in $A$.

Suppose that $K$ is permissible, $\mid \rightarrow_{n_{1}} K \cup\left(U_{1} \times\{A\}\right), \mapsto \rightarrow_{n_{2}} K \cup\left(U_{2} \times\{A\}\right)$ and that $U_{1} \cap U_{2}=\emptyset$. We shall deduce $\mid \rightarrow K$. We further suppose $\{\lambda \mid\langle\lambda, A\rangle \notin K\} \neq$ $\emptyset$; otherwise, $K$ contains $T \times\{A\}$ and so is basic, hence $\rightarrow K$. We put $\lambda_{0}=\min$ 
$\{\lambda \mid\langle\lambda, A\rangle \notin K\}$ and $K_{i}=K \cup\left(U_{i} \times\{A\}\right)$ for $i=1,2$.

By the induction hypothesis and by Proposition 6 we have the fact that

for every permissible matrix $L$ and for every formula $B$, if $\rightarrow L \cup\left(\mu^{\wedge} \times\{B\}\right)$ for every $\mu$ and if $d(B)<d(A)$, then $\rightarrow L$.

For the cut-free provable matrix $K_{i}(i=1,2)$, one of the following six cases occurs :

I. $K_{i}$ is basic.

II. $K_{i}$ is the conclusion of a left inference, and the principal matrix is contained in $K$.

III. $K_{i}$ is the conclusion of a left inference, but the principal matrix is not contained in $K$.

IV. $K_{i}$ is the conclusion of a right inference, the principal matrix is contained in $K$, but $\lambda_{0} \notin U_{i}$.

V. $K_{i}$ is the conclusion of a right inference, the principal matrix is contained in $K$, and $\lambda_{0} \in U_{i}$.

VI. $K_{i}$ is the conclusion of a right inference, but the principal matrix is not contained in $K$.

We shall show $\rightarrow K$ by cases.

CASE 1. Either $K_{1}$ or $K_{2}$ is of case I. We suppose, without loss of generality, that $K_{1}$ is the case. Then $T \times\{B\} \subseteq K_{1}$ for some $B$.

Subcase 1.1. $B$ is distinct from $A$. Then $T \times\{B\} \subseteq K$, so $K$ is basic, so $\mid \rightarrow$ $K$.

Subcase 1.2. Otherwise. If $\mu \in U_{2}$, then $\mu \notin U_{1}$ and so $\langle\mu, A\rangle \in K$; hence $U_{2} \times\{A\} \subseteq K$. So $K_{2}=K$, hence $\mid \rightarrow K$.

CASE 2. Either $K_{1}$ or $K_{2}$ is of case II. We suppose that $K_{1}$ is the case. Suppose that $\mid \rightarrow{ }_{<_{1}} K_{1} \cup\left(\nu_{1} \wedge \times\left\{B_{1}\right\}\right) \cup \cdots \cup\left(\nu_{h}{ }^{\wedge} \times\left\{B_{h}\right\}\right)$ for every $\nu_{1}, \cdots, \nu_{h}$ falsifying $g\left(\nu_{1}, \cdots, \nu_{h}\right) \leqq \nu$, and that $\nu^{-} \times\left\{g\left(B_{1}, \cdots, B_{h}\right)\right\} \subseteq K$. If $\rceil\left[g\left(\nu_{1}, \cdots, \nu_{h}\right) \leqq \nu\right]$, then

$$
\mapsto{ }_{<n_{1}} K \cup\left(\nu_{1}^{\wedge} \times\left\{B_{1}\right\}\right) \cup \cdots \cup\left(\nu_{h}^{\wedge} \times\left\{B_{h}\right\}\right) \cup\left(U_{1} \times\{A\}\right)
$$

and

$$
\mid \rightarrow{ }_{n_{2}} K \cup\left(\nu_{1}^{\wedge} \times\left\{B_{1}\right\}\right) \cup \cdots \cup\left(\nu_{h}^{\wedge} \times\left\{B_{h}\right\}\right) \cup\left(U_{2} \times\{A\}\right),
$$

so by the induction hypothesis $\rightarrow K \cup\left(\nu_{1} \wedge \times\left\{B_{1}\right\}\right) \cup \cdots \cup\left(\nu_{h}^{\wedge} \times\left\{B_{h}\right\}\right)$. Hence by the left inference $(g, \nu)^{-}$, we obtain $\rightarrow K$.

CASE 3. Either $K_{1}$ or $K_{2}$ is of case IV. We suppose that $K_{1}$ is the case. Suppose that $\rightarrow{ }_{<n_{1}}\left(K_{1}\right)^{\circ} \cup\left(\nu_{1}^{\wedge} \times\left\{B_{1}\right\}\right) \cup \cdots \cup\left(\nu_{h} \wedge \times\left\{B_{h}\right\}\right)$ for every $\nu_{1}, \cdots, \nu_{h}$ falsifying $\nu \leqq g\left(\nu_{1}, \cdots, \nu_{h}\right)$, and that $\nu^{+} \times\left\{g\left(B_{1}, \cdots, B_{h}\right)\right\} \subseteq K$ but $\lambda_{0} \oplus U_{1}$. Then $\rightarrow K^{\circ} \cup$ $\left(\nu_{1}^{\wedge} \times\left\{B_{1}\right\}\right) \cup \cdots \cup\left(\nu_{h}^{\wedge} \times\left\{B_{h}\right\}\right)$ for every $\nu_{1}, \cdots, \nu_{h}$ falsifying $\nu \leqq g\left(\nu_{1}, \cdots, \nu_{h}\right)$, since $\left(K_{1}\right)^{\circ}=K^{\circ}$. Hence by the right inference $(g, \nu)^{+}$, we obtain $\mapsto K$.

CASE 4. Both $K_{1}$ and $K_{2}$ are either of case III or of case V. We suppose that 
$K_{i}$ is of case III, and derive $\lambda_{0} \in U_{i}$; when $K_{i}$ is of case V, immediately $\lambda_{0} \in U_{i}$. Suppose that $\rightarrow{ }_{<n_{i}} K_{i} \cup\left(\mu_{1} \wedge \times\left\{A_{1}\right\}\right) \cup \cdots \cup\left(\mu_{k} \wedge \times\left\{A_{k}\right\}\right)$ for every $\mu_{1}, \cdots, \mu_{k}$ falsifying $f\left(\mu_{1}, \cdots, \mu_{k}\right) \leqq \mu$, and that $\mu^{-} \times\left\{f\left(A_{1}, \cdots, A_{k}\right)\right\}$ is contained in $K_{i}$ but is not in $K$. Then $f\left(A_{1}, \cdots, A_{k}\right)$ is identical with $A$ and so $\lambda_{0} \leqq \mu$, so $\left\langle\lambda_{0}, A\right\rangle \in \mu^{-} \times\left\{f\left(A_{1}, \cdots\right.\right.$, $\left.\left.A_{k}\right)\right\} \subseteq K_{i}$, so $\lambda_{0} \in U_{i}$. Hence, if $K_{i}$ is either of case III or of case V, then $\lambda_{0} \in U_{i}$. So, Case 4 does not occur since $U_{1} \cap U_{2}=\emptyset$.

CASE 5. One of $K_{1}$ and $K_{2}$ is of case III, while another of case VI. We suppose that $K_{1}$ is of case III, while $K_{2}$ of case VI. We also suppose that $A$ has the form $f\left(A_{1}, \cdots, A_{k}\right)$. Suppose that $\rightarrow<_{<n_{1}} K_{1} \cup\left(\mu_{1}^{\wedge} \times\left\{A_{1}\right\}\right) \cup \cdots \cup\left(\mu_{k} \wedge \times\left\{A_{k}\right\}\right)$ for every $\mu_{1}, \cdots, \mu_{k}$ falsifying $f\left(\mu_{1}, \cdots, \mu_{k}\right) \leqq \mu$, and that $\mu^{-} \times\{A\}$ is contained in $K_{1}$ but is not in $K$. Suppose further that $\rightarrow{ }_{<n_{2}}\left(K_{2}\right)^{\circ} \cup\left(\mu_{1} \wedge \times\left\{A_{1}\right\}\right) \cup \cdots \cup\left(\mu_{k} \wedge \times\left\{A_{k}\right\}\right)$ for every $\mu_{1}, \cdots, \mu_{k}$ falsifying $\nu \leqq f\left(\mu_{1}, \cdots, \mu_{k}\right)$, and that $\nu^{+} \times\{A\}$ is contained in $K_{2}$ but is not in $K$. By the argument given in Case 4 we have $\lambda_{0} \in U_{1}$ and $\lambda_{0} \leqq \mu$. Hence $\lambda_{0} \in U_{2}$ and so $\left(K_{2}\right)^{\circ}=K^{\circ}$.

SubCase 5.1. $\nu \leqq \mu$. Since the pair $\left(\nu, \lambda_{0}\right)$ has an upper bound $\mu$, it has the least upper bound $\nu \vee \lambda_{0}$. To show $\mapsto K$, it suffices to prove

$$
\begin{aligned}
& \mapsto K^{\circ} \cup\left(\mu_{1}^{\wedge} \times\left\{A_{1}\right\}\right) \cup \cdots \cup\left(\mu_{k} \wedge \times\left\{A_{k}\right\}\right) \text { for every } \\
& \mu_{1}, \cdots, \mu_{k} \text { falsifying } \nu \vee \lambda_{0} \leqq f\left(\mu_{1}, \cdots, \mu_{k}\right),
\end{aligned}
$$

and

$$
\left(\nu \vee \lambda_{0}\right)^{+} \times\{A\} \subseteq K,
$$

since from (4) in view of (5) we obtain $\mid \rightarrow K$ by the right inference $\left(f, \nu \vee \lambda_{0}\right)^{+}$.

For the proof of (4), suppose $\rceil\left[\nu \vee \lambda_{0} \leqq f\left(\mu_{1}, \cdots, \mu_{k}\right)\right]$. Then either $\rceil\left[\nu \leqq f\left(\mu_{1}\right.\right.$, $\left.\left.\cdots, \mu_{k}\right)\right]$ or $\rceil\left[\lambda_{0} \leqq f\left(\mu_{1}, \cdots, \mu_{k}\right)\right]$. We suppose $\rceil\left[\lambda_{0} \leqq f\left(\mu_{1}, \cdots, \mu_{k}\right)\right]$; otherwise, $\mid \rightarrow$ $K^{\circ} \cup\left(\mu_{1}^{\wedge} \times\left\{A_{1}\right\}\right) \cup \cdots \cup\left(\mu_{k} \wedge \times\left\{A_{k}\right\}\right)$ immediately. If $\rceil\left[f\left(\nu_{1}, \cdots, \nu_{k}\right) \leqq f\left(\mu_{1}, \cdots\right.\right.$, $\left.\left.\mu_{k}\right)\right]$, then $f\left(\nu_{1}, \cdots, \nu_{k}\right) \neq f\left(\mu_{1}, \cdots, \mu_{k}\right)$, so $\nu_{j} \neq \mu_{j}$ and so

$$
K^{\circ} \cup\left(\mu_{1}^{\wedge} \times\left\{A_{1}\right\}\right) \cup \cdots \cup\left(\mu_{k} \wedge \times\left\{A_{k}\right\}\right) \cup\left(\nu_{1}^{\wedge} \times\left\{A_{1}\right\}\right) \cup \cdots \cup\left(\nu_{k}^{\wedge} \times\left\{A_{k}\right\}\right)
$$

contains $T \times\left\{A_{j}\right\}$ for some $j(j=1, \cdots, k)$, hence it is basic and so is cut-free provable. Moreover, if $\lambda \leqq f\left(\mu_{1}, \cdots, \mu_{k}\right)$, then $\rceil\left(\lambda_{0} \leqq \lambda\right)$, so $\lambda^{-} \times\{A\} \subseteq K$, that is, $\langle\lambda, A\rangle \in K^{\circ}$; hence $f\left(\mu_{1}, \cdots, \mu_{k}\right)^{-} \times\{A\} \subseteq K^{\circ}$. Hence by the left inference $\left(f, f\left(\mu_{1}\right.\right.$, $\left.\left.\cdots, \mu_{k}\right)\right)^{-}$, we obtain $\rightarrow K^{\circ} \cup\left(\mu_{1}^{\wedge} \times\left\{A_{1}\right\}\right) \cup \cdots \cup\left(\mu_{k}^{\wedge} \times\left\{A_{k}\right\}\right)$.

Now we shall prove (5). We obtain $\left\langle\nu \vee \lambda_{0}, A\right\rangle \in\left(\mu^{-} \times\{A\}\right) \cap\left(\nu^{+} \times\{A\}\right) \subseteq$ $K_{1} \cap K_{2}=K$. So either $\left(\nu \vee \lambda_{0}\right)^{-} \times\{A\} \subseteq K$ or $\left(\nu \vee \lambda_{0}\right)^{+} \times\{A\} \subseteq K$, since $K$ is permissible. But $\rceil\left[\left(\nu \vee \lambda_{0}\right)^{-} \times\{A\} \subseteq K\right]$, since $\left\langle\lambda_{0}, A\right\rangle$ belongs to $\left(\nu \vee \lambda_{0}\right)^{-} \times\{A\}$ but does not to $K$. Hence $\left(\nu \vee \lambda_{0}\right)^{+} \times\{A\} \subseteq K$.

Subcase 5.2. Otherwise. To show $\rightarrow K$, it suffices to prove

$$
\mapsto K \cup\left(\mu_{1} \wedge \times\left\{A_{1}\right\}\right) \cup \cdots \cup\left(\mu_{k}^{\wedge} \times\left\{A_{k}\right\}\right) \text { for every } \mu_{1}, \cdots, \mu_{k} \text { in } T,
$$

since from (6) in view of the facts that $d\left(A_{1}\right), \cdots, d\left(A_{k}\right)<d(A)$ we obtain $\rightarrow K$ by 
the repeated use of $(3)$.

For the proof of (6), let $\mu_{1}, \cdots, \mu_{k}$ be given. Then either $\rceil\left[f\left(\mu_{1}, \cdots, \mu_{k}\right) \leqq \mu\right]$ or \rceil$\left[\nu \leqq f\left(\mu_{1}, \cdots, \mu_{k}\right)\right]$. If $\rceil\left[f\left(\mu_{1}, \cdots, \mu_{k}\right) \leqq \mu\right]$, then

$$
\mapsto<n_{1} K \cup\left(\mu_{1} \wedge \times\left\{A_{1}\right\}\right) \cup \cdots \cup\left(\mu_{k}^{\wedge} \times\left\{A_{k}\right\}\right) \cup\left(U_{1} \times\{A\}\right)
$$

and

$$
\mapsto{ }_{n_{2}} K \cup\left(\mu_{1}^{\wedge} \times\left\{A_{1}\right\}\right) \cup \cdots \cup\left(\mu_{k}^{\wedge} \times\left\{A_{k}\right\}\right) \cup\left(U_{2} \times\{A\}\right),
$$

so by the induction hypothesis, we obtain $\rightarrow K \cup\left(\mu_{1}^{\wedge} \times\left\{A_{1}\right\}\right) \cup \cdots \cup\left(\mu_{k} \wedge \times\left\{A_{k}\right\}\right)$. Supposing $\rceil\left[\nu \leqq f\left(\mu_{1}, \cdots, \mu_{k}\right)\right]$, we obtain the same result similarly.

CASE 6. One of $K_{1}$ and $K_{2}$ is of case V, while another of case VI. We suppose that $K_{1}$ is of case V, while $K_{2}$ of case VI. We also suppose that $A$ has the form $f\left(A_{1}\right.$, $\left.\cdots, A_{k}\right)$. Suppose that $\rightarrow<n_{1}\left(K_{1}\right)^{\circ} \cup\left(\nu_{1} \wedge \times\left\{B_{1}\right\}\right) \cup \cdots \cup\left(\nu_{h} \wedge \times\left\{B_{h}\right\}\right)$ for every $\nu_{1}, \cdots$, $\nu_{h}$ falsifying $\nu \leqq g\left(\nu_{1}, \cdots, \nu_{h}\right)$, and that $\nu^{+} \times\left\{g\left(B_{1}, \cdots, B_{h}\right)\right\} \subseteq K$ and $\lambda_{0} \in U_{1}$. Suppose further that $\mid \rightarrow{ }_{<n_{2}}\left(K_{2}\right)^{\circ} \cup\left(\mu_{1} \wedge \times\left\{A_{1}\right\}\right) \cup \cdots \cup\left(\mu_{k} \wedge \times\left\{A_{k}\right\}\right)$ for every $\mu_{1}, \cdots, \mu_{k}$ falsifying $\mu \leqq f\left(\mu_{1}, \cdots, \mu_{k}\right)$, and that $\mu^{+} \times\{A\}$ is contained in $K_{2}$ but is not in $K$. It follows $\lambda_{0} \notin U_{2}$, so $\left(K_{2}\right)^{\circ}=K^{\circ}$. To show $\rightarrow K$, it suffices to prove

$$
\begin{aligned}
& \mapsto K^{\circ} \cup\left(\nu_{1}^{\wedge} \times\left\{B_{1}\right\}\right) \cup \cdots \cup\left(\nu_{h}^{\wedge} \times\left\{B_{h}\right\}\right) \text { for every } \\
& \nu_{1}, \cdots, \nu_{h} \text { falsifying } \nu \leqq g\left(\nu_{1}, \cdots, \nu_{h}\right),
\end{aligned}
$$

since from (7) we obtain $\rightarrow K$ by the right inference $(g, \nu)^{+}$.

With a view to proving (7), suppose $\rceil\left[\nu \leqq g\left(\nu_{1}, \cdots, \nu_{h}\right)\right]$. If $\rceil\left[\mu \leqq f\left(\mu_{1}, \cdots\right.\right.$, $\left.\left.\mu_{k}\right)\right]$, then

$$
\begin{gathered}
\mapsto<n_{2}\left(K^{\circ} \cup\left(\nu_{1}^{\wedge} \times\left\{B_{1}\right\}\right) \cup \cdots \cup\left(\nu_{h}^{\wedge} \times\left\{B_{h}\right\}\right) \cup\left(\mu^{+} \times\{A\}\right)\right)^{\circ} \\
\cup\left(\mu_{1}^{\wedge} \times\left\{A_{1}\right\}\right) \cup \cdots \cup\left(\mu_{k} \wedge \times\left\{A_{k}\right\}\right),
\end{gathered}
$$

since

$$
\left(K_{2}\right)^{\circ}=K^{\circ}=\left(K^{\circ}\right)^{\circ} \subseteq\left(K^{\circ} \cup\left(\nu_{1}^{\wedge} \times\left\{B_{1}\right\}\right) \cup \cdots \cup\left(\nu_{h}^{\wedge} \times\left\{B_{h}\right\}\right) \cup\left(\mu^{+} \times\{A\}\right)\right)^{\circ} .
$$

Hence by the right inference $(f, \mu)^{+}$, we obtain

$$
\mapsto{ }_{n_{2}} K^{\circ} \cup\left(\nu_{1}^{\wedge} \times\left\{B_{1}\right\}\right) \cup \cdots \cup\left(\nu_{h}{ }^{\wedge} \times\left\{B_{h}\right\}\right) \cup\left(\mu^{+} \times\{A\}\right) .
$$

Now to show $\rightarrow K^{\circ} \cup\left(\nu_{1}^{\wedge} \times\left\{B_{1}\right\}\right) \cup \cdots \cup\left(\nu_{h}^{\wedge} \times\left\{B_{h}\right\}\right)$, it suffices to prove

$$
\left(K_{1}\right)^{\circ} \subseteq K^{\circ} \cup\left(\left(T-\mu^{+}\right) \times\{A\}\right),
$$

since from (8) it follows

$$
\mapsto<_{n_{1}} K^{\circ} \cup\left(\nu_{1} \wedge \times\left\{B_{1}\right\}\right) \cup \cdots \cup\left(\nu_{h} \wedge \times\left\{B_{h}\right\}\right) \cup\left(\left(T-\mu^{+}\right) \times\{A\}\right),
$$

from which and from the fact just proved, by the induction hypothesis we obtain $\mapsto$ $K^{\circ} \cup\left(\nu_{1}^{\wedge} \times\left\{B_{1}\right\}\right) \cup \cdots \cup\left(\nu_{h}^{\wedge} \times\left\{B_{h}\right\}\right)$.

To prove (8), it suffices to show that $\langle\lambda, A\rangle \in K^{\circ} \cup\left(\left(T-\mu^{+}\right) \times\{A\}\right)$ whenever $\langle\lambda, A\rangle \in\left(K_{1}\right)^{\circ}$. Suppose, contrary to the conclusion, that $\langle\lambda, A\rangle$ belongs to $\left(K_{1}\right)^{\circ}$ but does not to $K^{\circ} \cup\left(\left(T-\mu^{+}\right) \times\{A\}\right)$. Since $\langle\lambda, A\rangle \notin\left(T-\mu^{+}\right) \times\{A\}$ we have $\mu \leqq$ 
$\lambda$; since $\langle\lambda, A\rangle \notin K^{\circ}$, that is, $\rceil\left[\lambda^{-} \times\{A\} \subseteq K\right]$ we have $\lambda_{0} \leqq \lambda$. Hence the pair ( $\mu$, $\lambda_{0}$ ) has the least upper bound $\mu \vee \lambda_{0}$. We obtain $\left\langle\mu \vee \lambda_{0}, A\right\rangle \in\left(\lambda^{-} \times\{A\}\right) \cap\left(\mu^{+} \times\right.$ $\{A\}) \subseteq K_{1} \cap K_{2}=K$. So either $\left(\mu \vee \lambda_{0}\right)^{-} \times\{A\} \subseteq K$ or $\left(\mu \vee \lambda_{0}\right)^{+} \times\{A\} \subseteq K$ since $K$ is permissible. But if $\left(\mu \vee \lambda_{0}\right)^{-} \times\{A\} \subseteq K$, then $\left\langle\lambda_{0}, A\right\rangle \in\left(\mu \vee \lambda_{0}\right)^{-} \times\{A\} \subseteq K$, which is a contradiction. Now suppose $\left(\mu \vee \lambda_{0}\right)^{+} \times\{A\} \subseteq K$. Since $7\left[\mu^{+} \times\{A\} \subseteq K\right]$, we obtain $\mu \leqq \mu^{\prime}$, but $\left\langle\mu^{\prime}, A\right\rangle \notin K$ for some $\mu^{\prime}$. Then $\lambda_{0} \leqq \mu^{\prime}$, so $\mu \vee \lambda_{0} \leqq \mu^{\prime}$, so $\left\langle\mu^{\prime}, A\right\rangle$ $\in\left(\mu \vee \lambda_{0}\right)^{+} \times\{A\} \subseteq K$, which is also a contradiction. This completes the proof of (8) and hence of (7).

CASE 7. Both $K_{1}$ and $K_{2}$ are of case VI. We suppose, without loss of generality, that $\lambda_{0} \notin U_{2}$. We also suppose that $A$ has the form $f\left(A_{1}, \cdots, A_{k}\right)$. Suppose that $\mapsto{ }_{<n_{1}}\left(K_{1}\right)^{\circ} \cup\left(\mu_{1}^{\wedge} \times\left\{A_{1}\right\}\right) \cup \cdots \cup\left(\mu_{k}^{\wedge} \times\left\{A_{k}\right\}\right)$ for every $\mu_{1}, \cdots, \mu_{k}$ falsifying $\mu \leqq f\left(\mu_{1}\right.$, $\left.\cdots, \mu_{k}\right)$, and that $\mu^{+} \times\{A\}$ is contained in $K_{1}$ but is not in $K$. Suppose further that $\mapsto<n_{2}\left(K_{2}\right)^{\circ} \cup\left(\mu_{1} \wedge \times\left\{A_{1}\right\}\right) \cup \cdots \cup\left(\mu_{k}{ }^{\wedge} \times\left\{A_{k}\right\}\right)$ for every $\mu_{1}, \cdots, \mu_{k}$ falsifying $\nu \leqq f\left(\mu_{1}\right.$, $\left.\cdots, \mu_{k}\right)$, and that $\nu^{+} \times\{A\}$ is contained in $K_{2}$ but is not in $K$.

Subcase 7.1. The pair $(\mu, \nu)$ has an upper bound. Then the pair has the least upper bound $\mu \vee \nu$. To show $\rightarrow K$, it suffices to prove

$$
\begin{aligned}
& \mapsto K^{\circ} \cup\left(\mu_{1}^{\wedge} \times\left\{A_{1}\right\}\right) \cup \cdots \cup\left(\mu_{k}{ }^{\wedge} \times\left\{A_{k}\right\}\right) \text { for every } \\
& \mu_{1}, \cdots, \mu_{k} \text { falsifying } \mu \vee \nu \leqq f\left(\mu_{1}, \cdots, \mu_{k}\right),
\end{aligned}
$$

and

$$
(\mu \vee \nu)^{+} \times\{A\} \subseteq K
$$

since from (9) in view of (10) we obtain $\rightarrow K$ by the right inference $(f, \mu \vee \nu)^{+}$.

For the proof of (9), suppose $\rceil\left[\mu \vee \nu \leqq f\left(\mu_{1}, \cdots, \mu_{k}\right)\right]$. Then either $\rceil\left[\mu \leqq f\left(\mu_{1}\right.\right.$, $\left.\left.\cdots, \mu_{k}\right)\right]$ or $\rceil\left[\nu \leqq f\left(\mu_{1}, \cdots, \mu_{k}\right)\right]$. If $\rceil\left[\mu \leqq f\left(\mu_{1}, \cdots, \mu_{k}\right)\right]$, then we obtain $\rightarrow K^{\circ} \cup\left(\mu_{1}^{\wedge}\right.$ $\left.\times\left\{A_{1}\right\}\right) \cup \cdots \cup\left(\mu_{k} \wedge \times\left\{A_{k}\right\}\right)$ by the similar method as the proof of $(7) ;$ if $\rceil\left[\nu \leqq f\left(\mu_{1}\right.\right.$, $\left.\left.\cdots, \mu_{k}\right)\right]$, then we obtain the same result since $\left(K_{2}\right)^{\circ}=K^{\circ}$.

Now we shall prove (10). If $\mu \vee \nu \leqq \lambda$, then $\langle\lambda, A\rangle \in\left(\mu^{+} \times\{A\}\right) \cap\left(\nu^{+} \times\{A\}\right) \subseteq$ $K_{1} \cap K_{2}=K$. Hence $(\mu \vee \nu)^{+} \times\{A\} \subseteq K$.

Subcase 7.2. Otherwise. We obtain $\rightarrow K$ similarly to Subcase 5.2.

Thus we have proved Lemma, and hence the proof of Theorem is completed.

\section{Reference}

[1] M. Takano, Extending the family of intuitionistic many-valued logics introduced by Rousseau, this Annals, Vol. 7, No. 1 (1986), 47-56. 